\title{
Faire Verteilung der Leistungen und Mittel im Gesundheitswesen als ethische Herausforderung
}

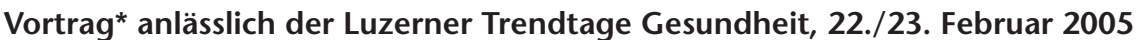

\section{R. Baumann-Hölzle}

\section{Ausgangslage}

Die Schere zwischen medizinischem und pflegerischem Bedarf, Anspruch und Angebotspalette auf der einen und dem Finanzierungswillen und den Finanzierungsmöglichkeiten auf der anderen Seite spreizt immer weiter auseinander. Als Reaktion auf diese Entwicklung bieten sich folgende Handlungsoptionen an: Rationalisierung, Erhöhung der Geldmenge, Reduktion von Bedarf und Angebot, verdeckte oder offene Rationierung. Sie werden einzeln reflektiert.

\section{Rationalisierung}

Nach wie vor steckt im Gesundheitswesen ein gewisses Rationalisierungspotential, indem Leistungen billiger erbracht und Mittel günstiger eingekauft werden, in gewissen Bereichen Personaleinsparungen möglich sind, grundsätzlich die Effizienz der zu erbringenden Leistungen gesteigert und auf sogenannte «futile treatments» verzichtet wird. Ein besonders hohes und nicht ausgeschöpftes Rationalisierungspotential liegt in einer verbesserten interdisziplinären und transdisziplinären Zusammenarbeit innerhalb der Ärzteschaft und mit den verschiedenen Berufsgruppen untereinander im Gesundheitswesen. Rationalisierungsmassnahmen vermindern die Behandlungs- und Betreuungsqualität nicht, sondern können sogar die Qualität der erbrachten Leistungen verbessern. Zudem erweitern sie die Handlungsmöglichkeiten für die Patientinnen und Patienten. Es ist denn auch eine moralische Pflicht, das Rationalisierungspotential im Gesundheitswesen auszuschöpfen, bevor Massnahmen ergriffen werden, die die Handlungsmöglichkeiten bei der Behandlung und Betreuung der Patienten einschränken.

Die Finanzierungsprobleme im Gesundheitswesen lassen sich jedoch nicht allein über Rationalisierungsmassnahmen lösen, denn es gibt viele Handlungen bei der Behandlung und Be- treuung von Menschen, die sich nur beschränkt rationalisieren lassen: Die Pflege zum Beispiel eines Menschen braucht Raum und Zeit, man kann einen Menschen nicht immer noch schneller waschen und ihm das Essen eingeben, und auch schwerkranke Menschen brauchen Zeit für die Entscheidungsfindung, bis sie wissen, was für Behandlungsmöglichkeiten sie in Anspruch nehmen wollen. $\mathrm{Zu}$ sehr sind sie durch ihre Krankheit verunsichert und von existentiellen Ängsten geplagt. Der Mensch mit seiner bedürftigen Leiblichkeit begrenzt die Rationalisierungsmöglichkeiten bei der Behandlung und Betreuung im Gesundheitswesen.

\section{Erhöhung der Geldmenge}

Die Geldmenge, die für Handlungen zur Verfügung steht, ist immer begrenzt. Das ist auch im Gesundheitswesen nicht anders. Die Krankenkassenprämien sind in der Schweiz heute derart hoch, dass bereits mehr als 40\% der Bevölkerung vom Staat Prämienverbilligungen erhält. Die finanzielle Belastung durch die Krankenkassenprämien übersteigt auch zunehmend die Finanzierungsmöglichkeit des Mittelstandes. Die Erhöhung der Geldmenge ist deshalb langfristig keine Lösungsmöglichkeit der Finanzierungsproblematik des Gesundheitswesens.

\section{Reduktion von Bedarf und Angebot}

Angesichts der offensichtlich begrenzten Finanzierungsmöglichkeiten des Gesundheitswesens können nicht jeder Bedarf und jedes Angebot finanziert werden. Es ist zwingend, eine Auswahl zu treffen. Die Diskussion darüber, welche Bedürfnisse und Angebote über die Grundversicherung zu finanzieren sind, ist unabdingbar. Um ethisch vertretbare Entscheide bezüglich Bedarf und Angebote fällen zu können, bedarf es jedoch der Daten- und Kostentransparenz, wie sie heute 
nicht vorhanden ist. Ohne eine solche transparente Datenlage können keine verantwortlichen Entscheide bezüglich der Leistungs- und Mittelverteilung im Gesundheitswesen getroffen werden. So lässt sich denn heute auch nicht entscheiden, inwieweit Bedarf und Angebot zu reduzieren sind oder nicht. $\mathrm{Zu}$ bedenken ist jedoch, dass die Conditio humana derart ist, dass der Behandlungs- und Betreuungsbedarf des Menschen als bedürftiges und verletzliches Wesen grenzenlos ist. Diese Unbegrenztheit stösst nun aber an die gesellschaftliche Finanzierungsgrenze, welche eine Grenzziehung bei Behandlung und Betreuung erzwingt. Diese Grenze kann nun entweder individuell und willkürlich als verdecke Rationierung oder öffentlich und transparent als demokratisch legitimierte Prioritätensetzung, ein anderes Wort für strukturelle Rationierung, gezogen werden.

\section{Verdeckte Rationierung}

Rationiert wird bereits heute, jedoch nicht transparent [1]. So findet in vielen Bereichen des schweizerischen Gesundheitswesens derzeit eine verdeckte Rationierung über Budgetbeschränkungen statt (Zugang zu Intensivpflegebetten, Assistenzärztestellen, Wartezeiten für Allgemeinpatienten usw.). Dadurch wird die Problematik der begrenzten Finanzierungsmöglichkeiten im Gesundheitswesen derzeit der Ärzteschaft und der Pflege bei ihren Entscheiden beim einzelnen Patienten zugespielt. Solch individuelle Entscheide führen jedoch zu Gerechtigkeitsproblemen, da jeder Arzt und jede Pflegende ihre Leistungen ohne faire Verteilungsregeln anders verteilt. Bereits heute beteiligt sich die öffentliche Hand unbegründet in wesentlich geringerem Mass an der Behandlung und Betreuung von chronischkranken Menschen. Diese Situation ist aus der Sicht des Patienten Entscheidungswillkür, die in einem demokratisch organisierten Staat nicht legitim ist. Da jedoch eine demokratisch abgestützte Prioritätensetzung fehlt - die Schweiz hat nicht einmal eine offizielle Gesundheitspolitik -, verschärft sich die Situation zunehmend und die eingangs genannte Schere geht immer weiter auseinander. Dadurch nimmt aber auch die verdeckte Rationierung zu.

\section{Keine Rationierung im Einzelfall}

Man kann es drehen und wenden wie man will, angesichts der beschränkten Finanzierungsmöglichkeiten und des begrenzten Finanzierungswil- lens kommt man in einem demokratisch organisierten Staat aus Gerechtigkeitsgründen nicht um eine Prioritätensetzung im Gesundheitswesen herum. Prioritätensetzungen sind bewusste Wertentscheide, die eine Auswahl zwischen Handlungsoptionen ermöglichen. Eine bewusste Wahl ist immer mit einem Verzicht auf andere Handlungsmöglichkeiten verbunden, was faktisch Rationierung bedeutet. Aufgrund des Anspruchs auf Gleichwertigkeit aller Menschen dürfen ihnen nicht im Einzelfall vorhandene notwendige Behandlungen und Betreuungen vorenthalten werden, sondern die Verteilung der Leistungen und Mittel im Gesundheitswesen hat innerhalb der Strukturen des Gesundheitswesens nach fairen und nach aussen transparenten Mechanismen zu geschehen. Eine faire Verteilung der Leistungen und Mittel im Gesundheitswesen ist nicht eine individuelle, sondern eine strukturelle Frage. Im folgenden werden denn auch die strukturellen Mechanismen des Gesundheitswesens auf ihre Wertsetzungen hin betrachtet.

\section{Das Gesundheitswesen als solidarisches System}

Betrachtet man das Gesundheitswesen unter dem Aspekt der Werte, so wird seine Grundproblematik deutlich: Das Gesundheitswesen ist als solidarisches System zur Behandlung und Betreuung von kranken Menschen organisiert. Inwieweit Krankheitsprävention dazugehört, wird bereits kontrovers debattiert. Grundsätzlich wird jedoch davon ausgegangen, dass kranke Menschen Anspruch auf Solidarität haben, unabhängig davon, ob sie mit ihrem Verhalten zur Krankheit beigetragen haben oder nicht. Diese Solidarität wird dem Menschen gewährt, bei dem die Behandlungs- und Betreuungsbedürfnisse von seiner Conditio humana her grenzenlos sind. Solange die Handlungsmöglichkeiten in Medizin und Pflege beschränkter waren, stand oft nur eine oder gar keine Behandlungsmöglichkeit zur Verfügung und so musste auch keine Wahl getroffen werden. Entsprechend reichten die vorhandenen finanziellen Mittel aus. Der medizinische Fortschritt hat nun zu einer enormen Mengenausweitung bei den Behandlungsund Betreuungsmöglichkeiten geführt. Diese allein schon führt zu Finanzierungsproblemen.

Auf dieses solidarische System des Gesundheitswesens treffen aber nicht nur kranke Menschen, die auf notwendige Behandlungen und Betreuung angewiesen sind, sondern auch Anbieter und Konsumenten, welche ihre eigenen 
Interessen maximieren möchten. Bei den Anbietern stösst das solidarische System des Gesundheitswesens mit demjenigen der Marktwirtschaft und ihrem inhärenten Impetus zur Profitmaximierung zusammen. Wird ein solidarisches System nicht vor überzogenen Eigen- oder Profitinteressen durch klare staatliche Spielregeln geschützt, so wird es ausgebeutet.

\section{Ausbeutung des Gesundheitswesens durch Anbieter und Konsumenten}

Die Medikamentenpreise sind denn auch in den letzten Jahren unverhältnismässig stark gestiegen und lassen sich in keiner Art und Weise weder mit ihrem Forschungsaufwand noch mit ihrem zusätzlichen Nutzen rechtfertigen. Dieser ist gegenüber herkömmlichen Medikamenten sehr oft marginal, wird aber zu übersetzten Preisen verkauft. Die Ärzteschaft wird von der Pharmaindustrie heftig umworben und steht vor allem in den Spitälern in mannigfaltigen Abhängigkeitsverhältnissen zur Industrie. In ähnlicher Art und Weise sind neue Abhängigkeiten der Universitäten durch Drittmittelfinanzierungen der Pharmaindustrie entstanden. Zusätzlich werden aber auch Patente auf Produkten erworben, die ursprünglich mit Geldern der öffentlichen Hand entwickelt worden sind und nun ebenfalls mit öffentlichen Geldern wiederum gekauft werden. Darüber hinaus wird die freie Marktwirtschaft unterlaufen, denn die pharmazeutische Industrie nimmt für sich zwar die Marktfreiheit in Anspruch, benutzt aber hierfür Gelder der öffentlichen Hand, von der sie für sich möglichst wenige Einschränkungen erwartet.

Gleichzeitig hat der Bedarf an Lifestylemedizin enorm zugenommen und medizinische Leistungen werden konsumiert. Tiefe Selbstbehalte bieten keinen Anreiz, sich gegenüber seiner Gesundheit selbstverantwortlich zu verhalten. Patienten, vor allem schwerkranke Patienten, sind sehr abhängig von der Beratung ihres Arztes. Entsprechend konsumieren sie, was ihnen der Arzt verschreibt.

Die Anbieter mit ihrer Logik der Gewinnmaximierung können so die im Prinzip grenzenlosen Ansprüche und Bedürfnisse des verletzlichen und immer bedürftigen Menschen gegenüber dem Gesundheitswesen aufnehmen und für ihren eigenen Profit nutzen. Die Bedürfnisse sind immer vorhanden, die Abnahme ist garantiert und die Finanzierung in jedem Fall durch die öffentliche Hand sichergestellt. Ein Traummarkt also, und entsprechend traumhaft hoch sind denn auch die Gewinne, die erzielt werden.
Mittlerweile rechtfertigt die Pharmaindustrie ihre hohen Gewinne auch damit, dass sie in der Schweiz ein wichtiger Arbeitgeber sei und mit ihren Steuern einen grossen Beitrag ans Bruttosozialprodukt leiste. Das Problem ist nur, dass dadurch andere Gesellschaftsbereiche, wie zum Beispiel derjenige der Bildung, der sehr viel mehr zur Gesundheit der Bevölkerung beitragen kann als die Pharmaindustrie, zunehmend weniger Geld zur Verfügung hat.

\section{Paradoxe Situationen}

Durch diese Vermischung des solidarischen mit dem wirtschaftlichen Wertsystem entstehen mannigfaltige Paradoxien:

Der Erfolg der Anbieter (Ärzte, Therapeuten, Institutionen usw.) besteht in einer Mengenausweitung ihrer Leistungen und gleichzeitig wird von ihnen verlangt, die Gesundheitskosten zu senken. Dies führt zum weiteren Paradox, dass die Anbieter des Gesundheitswesens eigentlich ein Interesse an möglichst vielen kranken Menschen haben müssen, denn nur über sie verdienen sie Geld. Entsprechend werden denn auch immer wieder neue Krankheitsgruppen generiert, wie z.B. der Mann und die Frau in der Menopause.

Entsprechend hat denn auch die Prävention, mit welcher kein Geld verdient werden kann, einen geringen Stellenwert: Statt Werbeverbote für Suchtmittel bezahlt die öffentliche Hand Therapieangebote, statt Koch- und Turnunterricht finanziert die Krankenkasse Xenical usw.

Die Ausbeutung des Gesundheitswesens wird durch sozialpolitische Gründe noch verstärkt: Das Gesundheits- und Sozialwesen ist der einzige Bereich, in der Menschen, die in einer funktionsorientierten Gesellschaft nicht mithalten können, einen Geborgenheitsraum finden.

\section{Funktionsanspruch der modernen Gesellschaft}

Optimale Funktionalität und Effizienz sind zentrale Werte der modernen Gesellschaft [2]. Dieses Paradigma hat den Menschen technischen Fortschritt, Unabhängigkeiten von Naturprozessen und neue Freiheiten gebracht. Diese Erfolgsstory hat aber auch ihre Schattenseiten. Im Zuge dieser Erfolge blendet der moderne Mensch seine existentielle Pflegebedürftigkeit aus. Er nimmt sich als sogenannt «autonomer» Mensch als völlig unabhängig wahr und verdrängt die abhängigen Seiten seines Mensch- 
seins. Eingeschränkte Funktionalität hat keinen Platz in seinem Lebensentwurf, und Pflegeaufgaben delegiert das Individuum immer mehr an den Staat.

Gleichzeitig steigen gesamtgesellschaftlich die Funktionsansprüche an das einzelne Individuum ständig. Raum und Zeit sind längst knappe Güter geworden. Den Menschen fehlt zunehmend die Zeit zur Pflege ihrer selbst, ihrer Beziehungen, ihrer Kinder, der Kranken, der Sterbenden und der Trauernden.

Nur «pflegeleichte» Menschen finden Platz im Arbeitsprozess. Die funktionale Gesellschaft selektioniert die Menschen nach dem Kriterium «survival of the fittest». Die «Fittesten» sind in diesem Kontext diejenigen Menschen, welche am wenigsten auf Pflege angewiesen sind und ihre abhängigen Seiten verdrängen können. Die Rationierung der Arbeitsplätze und die damit einhergehende Arbeitslosigkeit sind dabei unvermeidbar. Die ausselektionierten Menschen werden als «pflegebedürftig» aus dem Arbeitsprozess ausgeschieden. Und viele Menschen werden durch diesen Selektionsprozess auch tatsächlich krank. Es gehört zur Dynamik der funktionalen Gesellschaft, dass die Zahl der unterstützungsbedürftigen Menschen stetig zunimmt.

\section{Medizinalisierung von sozialen Problemen}

Dabei lässt sich eine Medizinalisierung der sozialen Probleme beobachten: Menschen, die den Funktionsansprüchen der Gesellschaft nicht gewachsen sind, werden krankgeschrieben und letztlich in die Invalidenversicherung abgeschoben. Es ist aber eine Tatsache, dass Armut krank macht.

Das Gesundheitswesen und die Invalidenversicherung sind bis anhin die Geborgenheitsräume der Gesellschaft, welche nach dem Solidar- und nicht nach dem Effizienzprinzip organisiert sind. Dies aber führt zu zweierlei Problemen: Zum einen werden Menschen, die durchaus arbeitsfähig wären, zwar finanziell unterstützt, jedoch von der Gesellschaft ausgegrenzt; und zum anderen müssen deshalb immer weniger Menschen für immer mehr Sozialhilfeempfänger aufkommen. Die jetzige Situation beginnt die Zahlungsfähigkeit und Zahlungsbereitschaft der Prämienzahlenden zu übersteigen. Wird die Situation rein aus finanzieller Sicht beurteilt, so findet tatsächlich ein Sozialausbau statt. Dieser ist aber ein Symptom für die darunterliegende Problematik des allgemeinen Rationalisierungsprozesses, der gesamtgesellschaft- lich zur Rationierung von Raum und Zeit führt, was faktisch einen Sozialabbau darstellt.

Es gehört zur Logik der funktionalen Gesellschaft, dass sie nun versucht, diese Probleme mit weiteren Effizienzsteigerungen in den Griff zu bekommen, und nun auch das Gesundheitswesen und die Invalidenversicherung nicht mehr nach solidarischen, sondern nach ökonomischen Kriterien zu organisieren versucht. Pflegebedürftigkeit und Krankheit werden in der Folge genauso zur Schuld wie Arbeitslosigkeit, d.h., sie sind persönlich zu verantworten und werden langfristig von der Gesellschaft nicht mehr solidarisch mitgetragen werden. Eine allein auf Funktionalität und Effizienz ausgerichtete Gesellschaft wird ohne Pflicht zur Solidarität und Fairness inhuman. Das Gesundheitswesen und die Invalidenversicherung können nicht die ganze soziale Verantwortung der Gesellschaft tragen. Auch die Wirtschaft hat eine soziale Verantwortung, indem sie Menschen mit geringerer Effizienzkapazität Arbeitsplätze zur Verfügung stellt.

\section{Nationale Gesundheitspolitik}

Hinter der Finanzierungsproblematik des Gesundheitswesens liegen also auch grundsätzliche gesellschafts- und sozialpolitische Probleme, welche mit einer systemischen Sichtweise zu betrachten und anzugehen sind. Im Vordergrund steht die Formulierung einer gesamtschweizerischen Gesundheitspolitik. Sie soll das Gesundheitswesen vor Ausbeutung sowohl durch die Anbieter als auch durch die Konsumenten schützen. Die finanziellen Exzesse der Pharmaindustrie sind zu unterbinden und es ist ihr ein angemesser Platz im Gesundheitswesen zuzuweisen: So schreibt Marcia Angell, die ehemalige Chefredaktorin des renommierten «New England Journal of Medicine»: «Despite all its excesses, this is an important industry that should be saved - mainly from itself. The public is dependent on it, and it should be made to carry out its original purpose of developing important drugs and selling them at reasonable prices. I have shown how the industry, corrupted by easy profits and greed, has deceived and exploited the American people.» [3]

\section{Demokratisch abgestützte Prioritäten- setzung (strukturelle Rationierung)}

Das Ausschöpfen des Rationalisierungspotentials und das Stoppen der Ausbeutung durch Anbieter und Konsumenten des schweizerischen 
Gesundheitswesens sind eine ethische Notwendigkeit. Diese Massnahmen entbinden jedoch nicht von einer Prioritätensetzung. Diese Wertentscheide treffen eine Wahl zwischen verschiedenen Handlungsoptionen. Damit ist immer auch ein Verzicht auf bestimmte Handlungen verbunden. Eine faire Verteilung der Leistungen und Mittel im Gesundheitswesen kommt um eine solche Grenzziehung nicht herum. Wird sie nicht vorgenommen, verstärkt sich die verdeckte Rationierung und damit die unfaire Verteilung der Leistungen und Mittel im Gesundheitswesen, wie wir sie heute schon antreffen. Die Leidtragenden von dieser «muddling through»-Gesundheitspolitik sind vor allem die chronischkranken, austherapierten Menschen. Sie haben Besseres verdient!

\section{Danksagung}

Für den Gedankenaustausch zur Ökonomisierung der Medizin danke ich Prof. Dr. Max Baumann, Küsnacht ZH.

\section{Literatur}

1 Die Ausführungen in diesem Abschnitt entstammen dem «Manifest für eine faire Mittelverteilung im Gesundheitswesen» der unabhängigen, interdisziplinären Arbeitsgruppe Gerechte Ressourcenverteilung im Gesundheitswesen. Beilage zur Schweizerischen Ärztezeitung Nr. 45/1999.

2 Die beiden folgenden Abschnitte sind aus Baumann-Hölzle R. Pflegebedarf als ethische Herausforderung; gegen ein einseitig funktionales Menschenbild. Neue Zürcher Zeitung, 9. März 2005, S. 15.

3 Angell M. The Truth about the Drug Companies. How they deceive us and what to do about it. New York: Random House; 2004.

\section{U ZER ER -T REN D T A G E - GESUNDHEIT}

\section{Luzerner Trendtage Gesundheit}

Donnerstag/Freitag, 23. und 24. März 2006

\section{Spitzenleistungen im Gesundheitswesen}

\section{Machbarkeit - Finanzierbarkeit - Ethik}

Themenschwerpunkte:

- Hochspezialisierte Medizin

- Rehabilitation - emerging market

- Möglichkeiten und Grenzen im Umgang mit Krebs

Informationen: Forum Gesundheit Luzern, Dr. Christof Wicki, Horwerstrasse 87, 6005 Luzern, Tel. 04131837 97, Fax 04131837 10, E-Mail: info@trendtage-gesundheit.ch, Website: www. trendtage-gesundheit.ch 